\title{
MMSE Changes During and After ECT in Late-Life Depression: A Prospective Study
}

\author{
Jasmien Obbels, M.Sc., Kristof Vansteelandt, Ph.D., Esmée Verwijk, Ph.D., \\ Annemieke Dols, M.D., Ph.D., Filip Bouckaert, M.D., Ph.D., \\ Mardien L. Oudega, M.D., Ph.D., Matbieu Vandenbulcke, M.D., Ph.D., \\ Max Stek, M.D., Ph.D., Pascal Sienaert, M.D., Ph.D.
}

\section{A R T I C L E I N F O}

Article bistory:

Received February, 272019

Revised April, 172019

Accepted April, 172019

Key Words:

ECT

late-life depression

cognition

\begin{abstract}
A B STRACT
Objective: There is ongoing concern about the impact of electroconvulsive therapy (ECT) on cognition in patients with late-life depression (LLD), especially in patients for whom pretreatment Mini-Mental State Exam (MMSE) scores are low. Our aim was to examine the evolution of cognitive effects of ECT, using the MMSE in a large group of patients with LLD. Methods: One bundred nine patients aged 55 years and older with unipolar depression, referred for ECT, were included in our study. The MMSE was assessed before, during, immediately after, and 6 months after ECT. Results: MMSE scores improved significantly during the course of ECT and remained stable during the 6-month period after ending ECT for the total group. In the group of patients with a low MMSE score (<24) at baseline, the MMSE score improved significantly during ECT, whereas in the group of patients with a normal MMSE score (24) at baseline, the score did not change significantly during ECT. In both groups, MMSE scores still increased slightly after ECT was discontinued. Conchsion: ECT does not cause deleterious cognitive effects, as measured with the MMSE, during and for 6 months after the ECT course in patients with LLD. In the event of a baseline cognitive impairment, MMSE scores tend to improve significantly during and for 6 months after the ECT course. The presence of pretreatment cognitive impairment should not lead clinicians to withbold ECT in older patients

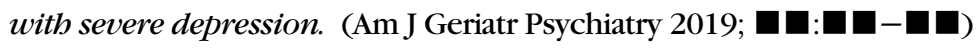

From the Academic Center for ECT and Neuromodulation (AcCENT) (JO, KV, FB, PS), University Psychiatric Center, KU Leuven-University of Leuven, Kortenberg, Belgium; Department of Medical Psychology (EV), Neuropsychology Department, Amsterdam UMC Academic Medical Center, The Netherlands; Department of Psychology (EV), Brain \& Cognition, University of Amsterdam, Amsterdam, The Netherlands; ECT Department (EV), Parnassia Psychiatric Institute, The Hague, The Netherlands; Department of Old Age Psychiatry (AD, MLO, MS), GGZ inGeest/Amsterdam University/VU Medical Center, Amsterdam Public Health Research Institute, Amsterdam Neuroscience, Amsterdam, The Netherlands; and the Old-Age Psychiatry (FB, MV), University Psychiatric Center, KU Leuven-University of Leuven, Kortenberg, Belgium. Send correspondence and reprint requests to Jasmien Obbels, Academic Center for ECT and Neuromodulation (AcCENT), University Psychiatric Center, KU Leuven-University of Leuven, Leuvensesteenweg 517, 3070 Kortenberg, Belgium. Phone: +32 27580731. Fax: +32 27595380.e-mail: jasmien.obbels@upckuleuven.be

(c) 2019 American Association for Geriatric Psychiatry. Published by Elsevier Inc. All rights reserved.

https:/ / doi.org/10.1016/j.jagp.2019.04.006 


\section{MMSE Changes During and After ECT in Late-Life Depression}

\section{INTRODUCTION}

$\mathrm{L}$ ate-life depression (LLD) is associated with an increased risk for developing physical morbidity and disability. ${ }^{1,2}$ The burden of depression on older patients, their caregivers, health services, and society is high. ${ }^{3,4}$ Electroconvulsive therapy (ECT) is an effective and well-established treatment for LLD. An older age predicts a better outcome with this treatment modality, and remission rates go up to $90 \%$ in patients over 65 years of age. ${ }^{5,6}$ However, there is ongoing concern about the possible impact of ECT on cognition, especially in the presence of pre-existing cognitive impairment and increasing age, implying that older patients might be more vulnerable than younger patients. ${ }^{7}$

Three reviews on the neuropsychological impact of ECT in patients with LLD concluded that the cognitive side effects of ECT are limited. ${ }^{8-10}$ Little is known on which factors are associated with the severity of the cognitive side effects of ECT. A bitemporal electrode placement has been associated with a higher degree of cognitive side effects, as compared to a unilateral placement. ${ }^{11,12}$ The impact of depression severity, the number of ECT sessions, age, and psychotic symptoms remains unclear. ${ }^{10,11,13-16}$

Most of the studies that have investigated the cognitive effects of ECT in patients with LLD have used the Mini-Mental State Exam (MMSE). ${ }^{10}$ This 30-item test is a screening instrument to assess global cognitive function. ${ }^{17}$ Although the MMSE is often criticized for having low specificity in detecting cognitive impairment in patients with $\mathrm{LLD}^{18-20}$ it is widely used in ECT trials and clinical settings.

Previous research has found that MMSE scores returned to pretreatment levels or even improved after ending an ECT course. ${ }^{10,21,22}$ Few studies, however, have reported the impact of ECT on MMSE scores during the treatment course itself. Two studies have reported a significant but transient decline in MMSE scores during ECT. ${ }^{21,23}$

It remains unclear whether cognitive status at baseline (pre-ECT) in older patients predicts cognitive effects during and after the ECT course. One study of 44 older patients with depression found that an intact pre-ECT cognitive status (as compared to an impaired baseline cognitive status) predicted a smaller decline of the MMSE score from baseline to 6 weeks after the last ECT treatment. ${ }^{24}$ Another study, however, found that patients with pretreatment cognitive dysfunction (as measured with the Mattis Dementia Rating Scale) experienced a larger cognitive improvement as compared to patients without pretreatment cognitive impairment. $^{25}$

Well-designed studies that used the MMSE for measuring cognitive effects of ECT in patients with LLD are sparse. Previous studies have often had a small sample size, variation in ECT treatment protocols and diagnosis, and no long-term cognitive follow-up after ending an ECT-course. ${ }^{8-10}$

In this study, we attempted to overcome these limitations by examining cognitive side effects of ECT with the MMSE in a large group of patients with LLD. Our aim was to map the evolution of MMSE scores before, during, immediately after, and 6 months after ECT. We compared a subgroup of patients with a low baseline MMSE score with a subgroup with a (close to) normal MMSE score.

\section{METHODS}

\section{Participants}

This naturalistic longitudinal study was conducted at two tertiary psychiatric hospitals (University Psychiatric Center, KU Leuven, Belgium, and GGZ inGeest, Amsterdam, The Netherlands). The study was part of the Mood Disorders in Elderly treated with ECT (MODECT) study, which investigated patients' clinical and structural brain characteristics and response to ECT. ${ }^{26}$ Patients aged 55 years and older with severe unipolar depressions according to the Diagnostic and Statistical Manual of Mental Disorders, Fourth Edition, Text Revision (DSMIV-TR) criteria $^{27}$ who were referred for ECT were included. Over a period of 3 years (2011-2013), 43 patients in Leuven and 67 patients in Amsterdam were recruited. Exclusion criteria were a comorbid major DSM-IV psychiatric illness or a (history of) neurologic illness (including stroke, dementia, and Parkinson disease). All participants gave their written informed consent. The protocol was approved by the Ethical Review Board of the VU Medical Center and by the Ethical Review Board of the Leuven University Hospitals. 


\section{ECT Procedure}

ECT was administered twice a week with a constant-current brief-pulse (0.5-1 msec) device (Thymatron System IV, Somatics, Lake Bluff, IL). Anesthesia was achieved with intravenous administration of etomidate $(0.2 \mathrm{mg} / \mathrm{kg})$ and succinylcholine $(1 \mathrm{mg} / \mathrm{kg})$. Patients were started on a course of ECT with right unilateral (RUL) electrode (d'Elia) placement. At the first treatment, the patient's seizure threshold (ST) was established by empirical titration. Subsequent treatments were given at 6 times the ST. Patients were treated until remission-defined as a Montgomery-Åsberg Depression Rating Scale (MADRS) ${ }^{28}$ score of less than 10 at two consecutive weekly ratings-or until patients showed no further improvement during the last 2 weeks of ECT. Patients were switched to bilateral (BL) ECT at 1.5 times the ST when, after six unilateral treatments, there was no clinical improvement. BL ECT was started immediately when the involved experienced geriatric psychiatrist concluded that the patient's clinical condition was too severe and life threatening (evidenced by increased suicidality, dehydration or severe weight loss, or debilitating psychotic features). Psychotropic medication was discontinued at least 1 week prior to ECT or, if discontinuation was deemed impossible, was kept stable from 6 weeks prior to ECT through the ECT course.

\section{Assessments \\ Clinical assessment}

Diagnosis of depression according to DSM-IV criteria $^{27}$ was made by experienced geriatric psychiatrists and confirmed by the Mini-International Neuropsychiatric Interview. ${ }^{29}$ Medication use and demographic variables were obtained by interview. The MADRS was used to assess the depressive symptom severity 1 week prior to ECT, weekly during ECT, after the sixth ECT course, and 1 week, 4 weeks, and 6 months after the last ECT course.

\section{Neuropsychological assessment}

The MMSE was used to assess cognitive functioning. An extensive neuropsychological battery was performed before the first ECT treatment and 6 months after the last ECT treatment, the results of which are discussed extensively elsewhere. ${ }^{30}$ The MMSE is a global cognitive screening instrument to assess orientation, attention, executive functioning, and memory. ${ }^{17}$ A clinical neuropsychologist, trained research assistant, or a supervised psychologist/psychiatrist in training assessed the cognitive functioning of ECT patients 1 week prior to ECT, weekly during ECT, and 1 week, 4 weeks, and 6 months after the last ECT treatment. Although the study plan was to have a final assessment 6 months after the ECT index course, it was not always possible to assess patients exactly 6 months after ECT due to practical circumstances. Nevertheless, we made great effort to reassess the patients, even if the time of reassessment was later than the intended 6 months after the ECT index course. Therefore, while the majority of patients had a final assessment around 6 months after the ECT index course, a limited number of patients had their final assessment beyond this time point. Traditionally, a MMSE cutoff score of 24 has been used to indicate cognitive impairment. ${ }^{31}$ We therefore assigned patients with a baseline MMSE score below 24 to a cognitive-impaired group and patients with a baseline score of 24 or above to a cognitive-unimpaired group.

\section{Statistical Analysis}

To examine cognitive functioning during and after ECT treatment, we estimated linear mixed models $(\mathrm{LMMs})^{32}$ with repeated measurements of the MMSE (Level 1) being nested within patients (Level 2). As ECT treatment may directly affect cognitive functioning during ECT treatment, we assumed cognitive change might be different during ECT treatment compared to at follow-up after ECT treatment. Therefore, piecewise random LMMs were estimated with a twopiece linear function of time corresponding to the trends during ECT treatment and the trends after ECT treatment. ${ }^{33}$ Such a model assumes that the MMSE is linearly related to time but allows for the (linear) trend during ECT treatment to be different from the trend after the ECT treatment. Because of the inclusion of random effect in the model, patients may show patient-specific trends that differ before and after ECT. For more information on piecewise LMMs, we refer the reader to Naumova et al. ${ }^{33}$ Four piecewise LMMs were estimated. First, we estimated a model for the total sample without covariates. Next, 


\section{MMSE Changes During and After ECT in Late-Life Depression}

we added covariates to examine the effects of being psychotic or not, electrode position (RUL versus BL, or switched from RUL to BL), depression at baseline (MADRS score), age, and the number of times the MMSE was administered (to adjust for learning effects). Next, a similar model was estimated but with an additional group variable that indicated whether patients belonged to a cognitive-impaired group at baseline (MMSE <24) or a cognitive-unimpaired group at baseline (MMSE $\geq 24$ ). Finally, in a fourth model, we examined the effects of the covariates mentioned above by adding them to the previous model. In the analyses with covariates, the continuous variables of age, MADRS score at baseline, and number of MMSEs administered were mean-centered. Note that the MMSE was assessed weekly, and consequently, the number of MMSE assessments correlated highly with the number of ECT treatments $(r=0.58$, $\mathrm{N}=109, \mathrm{p}<0.0001)$. For reasons of multicollinearity, we decided not to include number of ECT treatments as a covariate in these models (see Results section for more detailed information on the effects of number of ECT treatments on MMSE trajectories and the rationale at the basis of this decision). In line with recommendations of Verbeke and Molenberghs, ${ }^{32}$ Wald test scores with Kenward-Rogers degrees of freedom were used for inference. Further, with respect to missing data, note that inference for the proposed models is valid under the assumption of missingness at random. ${ }^{32,34}$ Finally, an effect size (ES) was calculated in the total group by means of a pre/post standardized mean gain score ${ }^{35,36}$ using model-based means. The interpretation of this ES is the same as that for Cohen's $d,{ }^{37}$ that is, $\mathrm{d} \leq 0.20$ is considered a small ES, $\mathrm{d} \approx 0.50$ a medium $\mathrm{ES}$, and $\mathrm{d} \geq 0.80$ a large ES. We refrained from calculating ESs for group differences at endpoint because the groups were not randomized and the meaning of such an ES is unclear, as mean differences at endpoint may be the result of differences in initial status at baseline, differences in growth during and after ECT, or both. All models were estimated using SAS version 9.3 (SAS Institute, Cary, NC). ${ }^{38}$

\section{RESULTS}

\section{Clinical and Demographic Characteristics}

Figure 1 presents a flow chart of the patient selection. Clinical and demographic characteristics of the

\begin{tabular}{lc}
\hline TABLE 1. Demographic and Clinical Characteristics $(\mathbf{N}=\mathbf{1 0 9})$ \\
\hline Mean age, y (SD) & $73.1(8.4)$ \\
Gender, female, N (\%) & $72(66.1)$ \\
DSM-IV diagnosis, N (\%) & \\
$\quad$ Major depressive disorder & $55(50.5)$ \\
$\quad$ Major depressive disorder with psychosis & $54(49.5)$ \\
MADRS, mean (SD) & \\
Baseline & $33.4(8.8)$ \\
6 months post-ECT & $10.7(9.7)$ \\
MMSE, mean (SD) & \\
Baseline & $24.3(5.1)$ \\
6 months post-ECT & $27.4(3.2)$ \\
Number of ECT treatments, mean (SD) & $14.3(8.0)$ \\
Electrode position, N (\%) & \\
$\quad$ Unilateral & $66(60.6)$ \\
Bilateral & $8(7.3)$ \\
Switch from unilateral to bilateral & $35(32.1)$
\end{tabular}

Notes: DSM-IV: Diagnostic and Statistical Manual of Mental Disorders, Fourth Edition; ECT: electroconvulsive therapy; MADRS: Montgomery-Åsberg Depression Rating Scale; MMSE: Mini-Mental State Exam; SD: standard deviation.

109 patients who were included in the study are shown in Table 1. Patients had a mean age of 73.1 years (standard deviation $[\mathrm{SD}]=8.4$ ). The sample consisted of 72 women (66.1\%) and 37 men. Fiftyfive patients $(50.5 \%)$ had a DSM-IV diagnosis of a major depressive disorder with psychotic features. The mean MADRS score decreased significantly from $33.4(\mathrm{SD}=8.8)$ at baseline to $10.7(\mathrm{SD}=9.7)$ at the 6-months post-ECT interval $(\mathrm{t}[82]=14.98, \mathrm{p}<0.0001)$. The mean total number of ECT sessions at the 6-month interval was $14.3(\mathrm{SD}=8)$. In 66 patients $(60.6 \%)$, RUL ECT was used, and in 8 patients $(7.3 \%)$ BL ECT was used. Thirty-five patients (32.1\%) were switched during their ECT course from RUL to BL ECT. Fifteen patients $(14.4 \%)$ had maintenance ECT during the 6-month time interval after the index treatment. We refer the reader to the article of Dols et al. ${ }^{26}$ for a more detailed description of the demographics and clinical characteristics of the sample.

\section{Neuropsychological Assessment}

MMSE during and after ECT treatment in the total group

At baseline, patients had on average a total MMSE score of 24.29 (standard error [SE] $=0.52 ; 95 \%$ confidence interval [CI]: 23.27-25.31). The MMSE score, on average, increased significantly by 1.37 points per month (SE $=0.26$; 95\% CI: 0.85-1.88) during the ECT course (main effect time [piece 1]: $F[1,87]=27.83$, 
FIGURE 1. Flow chart of the patient selection.

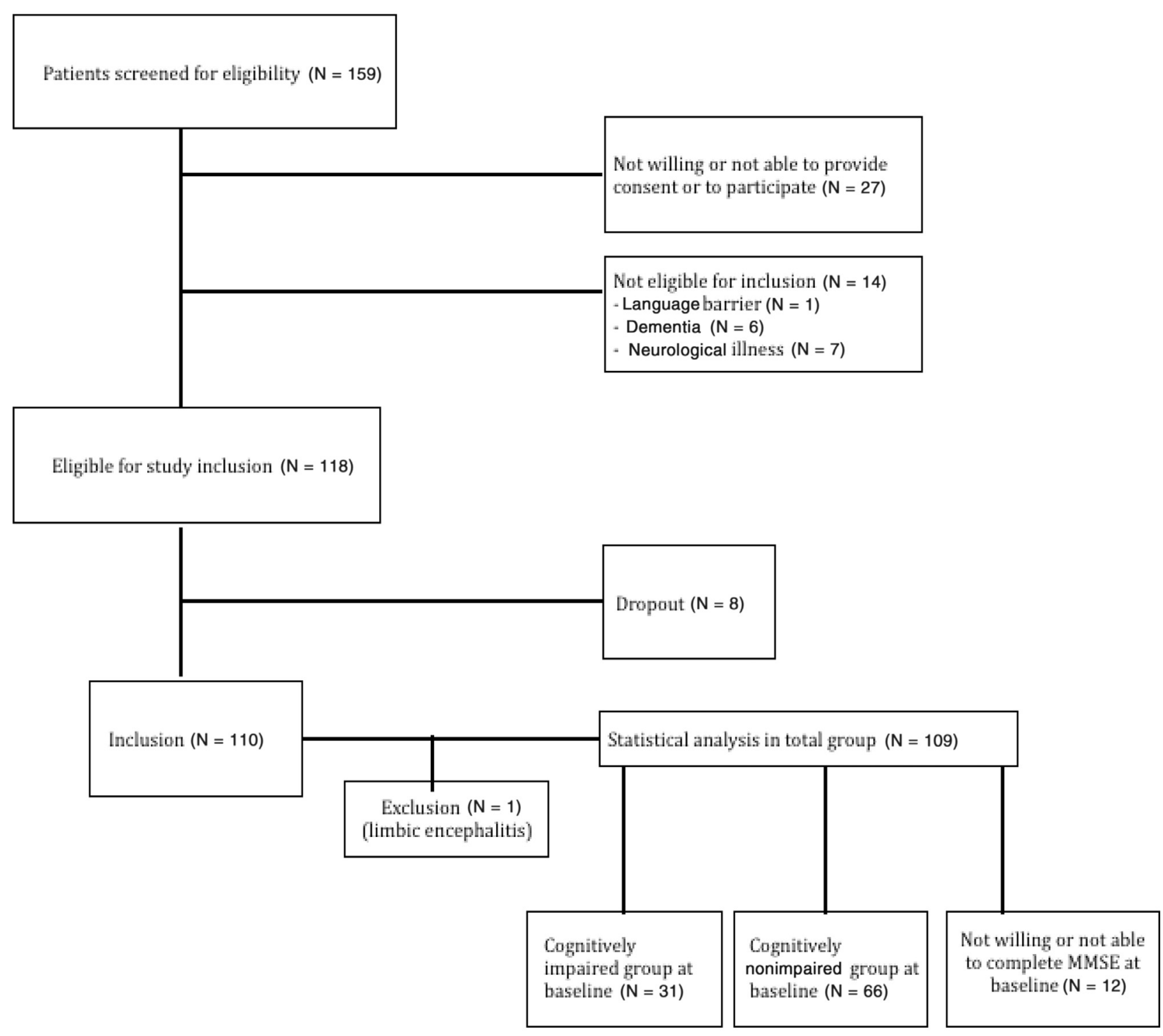

$\mathrm{p}<0.0001$; change between baseline and end of index course: ES $=0.44$ ), and patients had, on average, an MMSE score of 26.68 (SE $=0.37: 95 \%$ CI: 25.96 $-27.41)$ at the end of the treatment. However, during the follow-up period, the MMSE score remained stable on average (rate of change per month $=0.10$ [SE $=$ 0.06 ; $95 \%$ CI: -0.03 to 0.23 ]; main effect time [piece $2]: F[1,118]=2.34, p=0.13)$. The change of the MMSE during and after ECT is shown in Figure 2.

In a next step, we examined the effect of a number of covariates. The results of this analysis showed significant psychosis by time (piece 1) interaction implying a significant difference in MMSE change during the course of ECT for patients with a psychotic depression versus patients with a nonpsychotic depression $(F[1,107]=5.21, p=0.02)$. In particular, patients with a psychotic depression showed a significant increase in MMSE scores (rate of change per month $=1.91$ [SE $=0.42 ; 95 \%$ CI: 1.07-2.74]; $\mathrm{t}[105]=4.53, \mathrm{p}=0.0001$ ), while patients with a nonpsychotic depression remained stable during the ECT treatment (rate of change per month $=0.62$ 


\section{MMSE Changes During and After ECT in Late-Life Depression}

\section{FIGURE 2. MMSE during and after ECT in the total group}

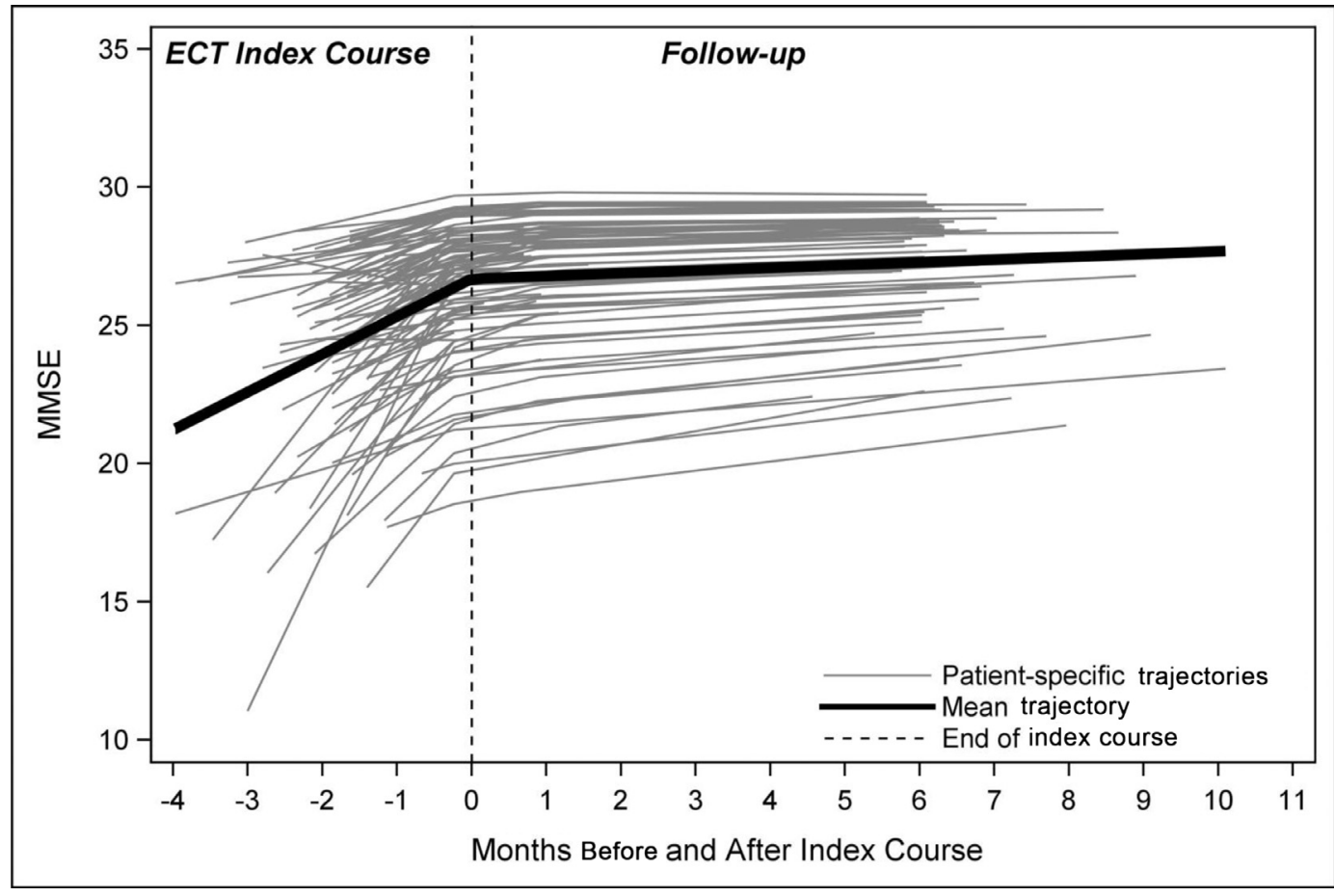

$[\mathrm{SE}=0.39 ; 95 \%$ CI: -0.14 to 1.39$] ; \mathrm{t}[135]=1.61$, $\mathrm{p}=0.11$ ). As can be seen in Figure 3, patients with a psychotic depression scored lower at baseline on the MMSE compared to patients with a nonpsychotic depression (main effect psychosis at baseline: $\mathrm{F}$ $[1,95]=6.31, p=0.01)$. Both of these groups did not differ significantly from one another after the ECT treatment (psychosis by time [piece 2] interaction: $\mathrm{F}[1,183]=1.31, \mathrm{p}=0.25)$ and remained stable on the MMSE after the ECT treatment (main effect time [piece 2]: $\mathrm{F}[1,198]=3.39, \mathrm{p}=0.07)$. In addition, at baseline, during, as well as after the ECT treatment, older patients scored lower on the MMSE compared to younger patients (main effect age: $\mathrm{F}$ $[1,140]=21.76, p<0.0001)$. Finally, patients who were tested more frequently on the MSSE scored higher on the MMSE than patients who were tested less frequently on the MMSE, which may point to learning effects (main effect number of times MMSE was administered: $F[1,140]=6.87, p=0.01$ ). We also estimated a model with number of ECT treatments instead of number of MMSEs administered.
Patients who received more ECT treatments scored significantly higher on the MMSE (main effect number of ECT treatments: $F[1,95.9]=5.09, p=0.03$ ). In a model with both predictors-number of MMSEs administered and number of ECT treatments-both predictors were not significant anymore (probably due to their high correlation). Therefore, we decided to retain only the number of MMSEs administered in the model. Note that these results indicate that patients who received more ECT treatments were characterized by less cognitive impairment as measured by the MMSE. Finally, no effects of electrode position (main effect electrode position: $\mathrm{F}[1,135]=1.16, \mathrm{p}=0.28$; electrode position by time [piece 1] interaction: $F[1,96.9]=0.00$, $\mathrm{p}=0.96$; electrode position by time [piece 2] interaction: $F[1,183]=0.15, p=0.70)$ and severity of depression (MADRS score) at baseline were found (main effect MADRS: $\mathrm{F}[1,135]=0.24, \mathrm{p}=0.63$; MADRS score by time [piece 1] interaction: $F$ $[1,95.5]=0.64, \mathrm{p}=0.42 ;$ MADRS score by time [piece 2] interaction: $F[1,189]=0.00, \mathrm{p}=0.97$ ). 
FIGURE 3. MMSE during and after ECT in a group of patients with and without psychotic features.

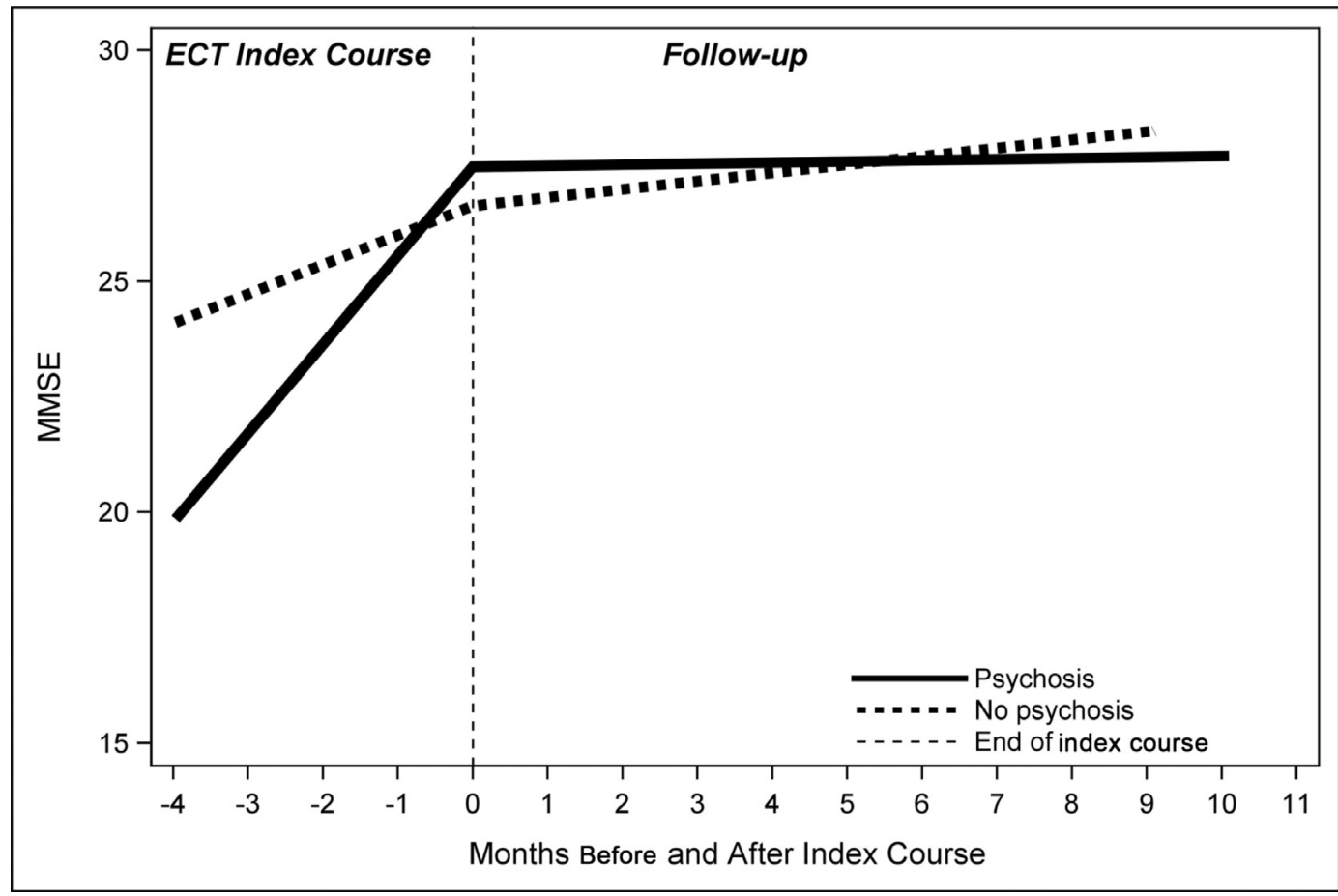

MMSE during and after ECT treatment in the group of cognitively impaired (MMSE <24) and not impaired (MMSE 24) patients at baseline

As shown in Figure 4, the change in MMSE score during the course of ECT treatment differed significantly between the group with baseline cognitive impairment $(\mathrm{N}=31)$ and the group with baseline normal MMSE, or no cognitive impairment $(\mathrm{N}=66)$ (group by time [piece 1] interaction: $F[1,75.1]=34.20$, $\mathrm{p}<0.0001)$. While the MMSE score in the group with a low baseline MMSE score increased significantly over the course of ECT (rate of change per month $=2.79$ [SD $=0.35]$; 95\% CI: 2.08-3.50; $\mathrm{t}[72.3]=7.87, \mathrm{p}<0.0001)$, the MMSE-score in the group without cognitive impairment remained unchanged during the ECT course (rate of change per month $=0.26$ [SD $=0.25]$; $95 \%$ CI: -0.23 to 0.76 ; $\mathrm{t}[81.4]=1.07, \mathrm{p}=0.29$ ). Moreover, MMSE scores of both groups showed a similar small but significant increase after ending ECT (main effect time [piece 2]: $\mathrm{F}[1,100]=4.22, \mathrm{p}=0.04)$; group by time [piece 2] interaction: $\mathrm{F}[1,100]=0.18, \mathrm{p}=0.67)$.
In a next step, we examined the effect of a number of covariates. The results of this analysis revealed again a significant difference between the groups with low and high cognitive performance at baseline (group by time [piece 1] interaction: F[1,76.7] = 31.35, $\mathrm{p}<0.0001)$. The MSSE scores of the group with low cognitive performance at baseline increased significantly during ECT treatment (rate of change $=2.83$ $[\mathrm{SD}=0.39] ; \quad 95 \% \quad \mathrm{CI}: \quad 2.06-3.60 ; \quad \mathrm{t}[7.30]=7.30$, $\mathrm{p}<0.0001$ ), while those of the group with high cognitive performance at baseline remained stable during ECT treatment (rate of change $=0.29$ [SD $=0.27$ ); $95 \% \mathrm{CI}:-0.25$ to $0.82 ; \mathrm{t}[99.6]=1.07, \mathrm{p}=0.29)$. Moreover, MMSE scores of both groups showed a similar small but significant increase after ending ECT (main effect time [piece 2]: $\mathrm{F}[1,87.7]=4.46, \mathrm{p}=0.04$; group by time [piece 2] interaction: $F[1,102]=0.00$, $\mathrm{p}=0.96$ ). Contrary to the analysis with the total group, patients with a psychotic depression versus patients with a nonpsychotic depression did not differ significantly either during the ECT course (psychosis by time [piece 1] interaction: $F[1,87.6]=2.92$, $\mathrm{p}=0.09$ ) or after ending ECT (psychosis by time 


\section{MMSE Changes During and After ECT in Late-Life Depression}

FIGURE 4. MMSE during and after ECT in the group of cognitively impaired (MMSE <24) and nonimpaired (MMSE $>=24)$ patients at baseline.

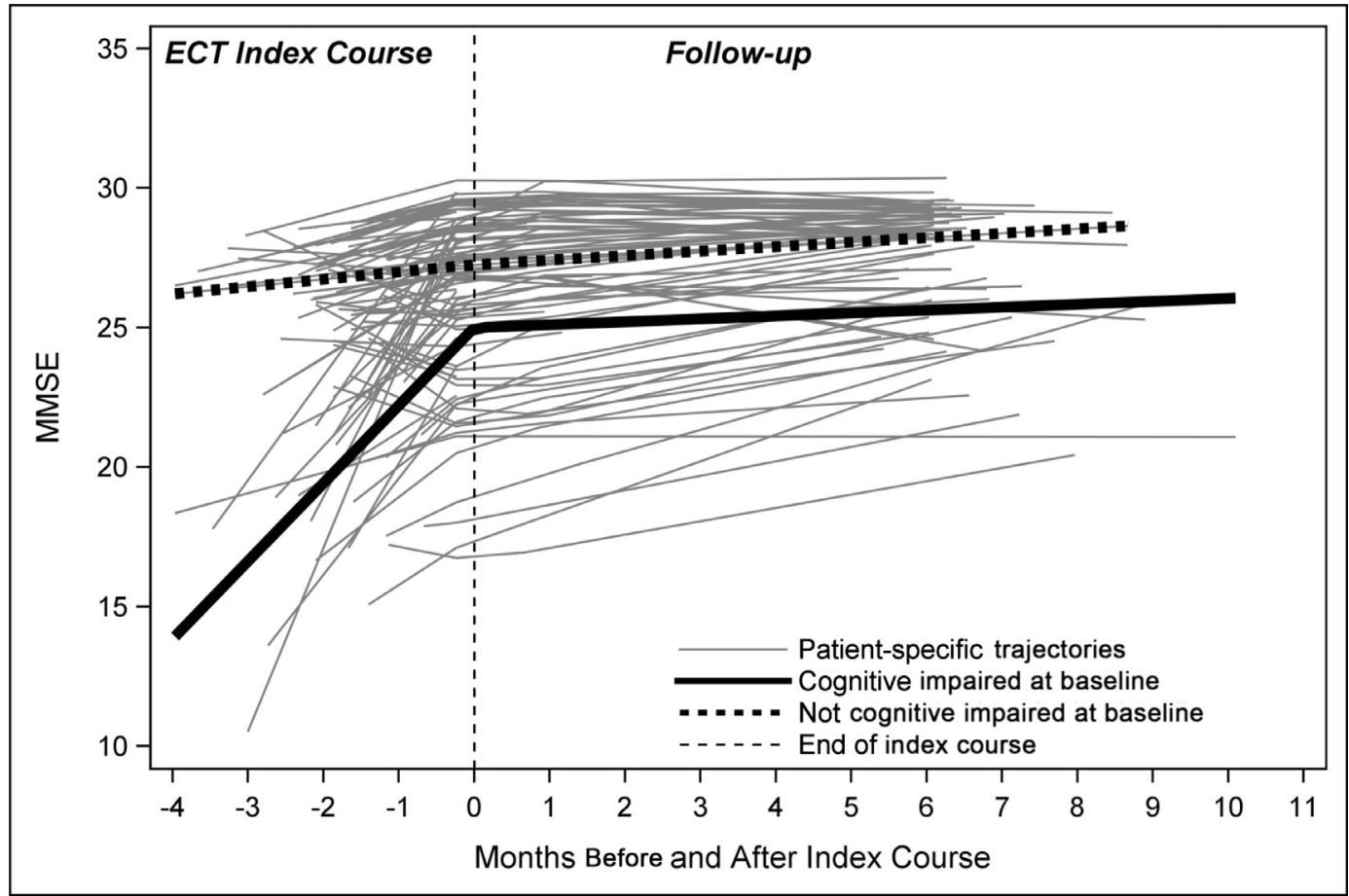

[piece 2] interaction: $\mathrm{F}[1,96.3]=2.31, \mathrm{p}=0.13$ ). Finally, in line with the results in the total group, older patients scored lower on the MMSE during as well as after ECT treatment (main effect age: $\mathrm{F}$ $[1,88.4]=17.53, \mathrm{p}<0.0001)$, and patients who were assessed more frequently on the MMSE scored higher on the MMSE (main effect number of times MMSE was administered: $F[1,91]=4.16, p=0.04)$. Further, we also estimated a model with number of ECT treatments instead of number of MMSEs administered. However, there was no effect of number of ECT treatments (main effect number of ECT treatments, $F[1,93.5]=2.70, p=0.10)$. Once again, both predictors-number of MMSEs administered and number of ECT sessions-were not significant anymore in a model including both predictors simultaneously (probably due to their high correlation). Therefore, we opted for a model with only number of MMSEs administered. From these results, it may be clear that patients who received more ECT treatments were not characterized by more cognitive impairment as measured by the MMSE.
Finally, no effects of electrode position (main effect electrode position: $\mathrm{F}[1,88.4]=1.99, \mathrm{p}=0.16$; electrode position by time [piece 1] interaction: $\mathrm{F}[1,77.4]=0.25$, $\mathrm{p}=0.62$; electrode position by time [piece 2] interaction: $F[1,95.8]=0.25, p=0.62$ ) and severity of depression (MADRS score) at baseline were found (main effect MADRS score: $\mathrm{F}[1,85.2]=0.42, \mathrm{p}=0.52$; MADRS score by time [piece 1] interaction: $F$ $[1,67.2]=0.00, p=0.98$; MADRS score by time [piece 2] interaction: $F[1,99.6]=0.17, p=0.68)$.

\section{DISCUSSION}

In patients with LLD, MMSE scores showed a significant improvement during an index course of ECT. This result is in sharp contrast with other studies that reported a significant decline in MMSE scores during an ECT course. $^{21,23}$ The differences in findings between our study and other studies could be due to differences in sample characteristics and/or treatment protocols. For example, Rubin et al. ${ }^{21}$ found a 
cognitive decline in MMSE scores of about three points at approximately two-thirds of the way through an ECT course. However, their findings were based on a small and heterogeneous group of patients ( $\mathrm{N}=48$, unipolar or bipolar), of whom $35 \%$ had a history of stroke and/or Parkinson disease. Moreover, ECT was administered three times a week, and 75\% of their patients were treated with a BL electrode position. Oudega et al. ${ }^{23}$ also reported a cognitive decline of MMSE scores during the ECT course. However, their analyses were based on the lowest mean MMSE scores during ECT.

In our study, after the initial improvement, MMSE scores remained unchanged during a 6-month follow-up period, in line with findings reported in other studies. ${ }^{10,13,30}$

Surprisingly, patients with a low MMSE score at baseline improved significantly during the ECT course, whereas a normal MMSE score at baseline did not change significantly. Patients with a cognitive impairment before the start of ECT seemed to catch up, during the course of ECT, with patients who had a normal cognitive function. In the months after ECT, cognitive function further slightly but significantly improved in both groups. Our findings contradict the assumption that older patients with an impaired cognitive function before the start of ECT, as measured with the MMSE, are more vulnerable for developing ECT-related cognitive side effects. ${ }^{7,24}$ On the contrary, our data suggest the majority of older patients with a low baseline MMSE score will tolerate the treatment course. However, our results need to be interpreted with caution. It could be that the improvement in cognition among the group with low MMSE scores at baseline could be due to the fact that, starting with a low MMSE score, there is more room to practice effects.

Apart from pretreatment cognitive status, the presence of psychotic symptoms also had an impact on the evolution of cognitive function. Patients with a psychotic depression showed a significant increase in MMSE scores during the ECT course, whereas MMSE scores of patients without psychotic symptoms did not change significantly. This differential effect seemed to wane: Six months after ECT, MMSE scores of patients with or without psychotic symptoms did not differ. This seems to be in line with previous research showing that, in patients with psychotic depression, cognitive performance does not worsen or even improves after ECT. ${ }^{16,39}$
Counterintuitively, we, as others, ${ }^{13,24,30,40}$ did not find an association between neurocognitive performance and change in depression severity.

MMSE is one of the most widely used cognitive tests but, simultaneously, is often maligned. Most of the available research on cognitive effects of ECT has relied on this rather simple screening tool. Of 84 studies meta-analyzed by Semkovska and McLoughlin, ${ }^{11} 30$ used the MMSE. The MMSE, nevertheless, has a number of drawbacks. It provides a limited screening of specific neuropsychological functions and may lack the sensitivity to detect subtle cognitive changes induced by ECT or LLD. ${ }^{18,41}$ Repeated testing might lead to ceiling and test-retest effects. ${ }^{8}$ In our study, patients with a higher MMSE score at the end of the treatment course had been tested more frequently than those with a lower MMSE score, suggesting a possible learning effect. Moreover, the change in MMSE score during the ECT course was related to the number of MMSE tests that patients had performed. This is probably due to learning effects. Therefore, our results need to be interpreted with caution. However, the fact that patients experienced learning effects supports the idea that ECT is not detrimental to cognition as measured with the MMSE.

There is a need for easy-to-use bedside neuropsychological tests that are specifically designed to detect cognitive effects of ECT. Monitoring these cognitive effects is a complex matter that should take a wide variety of parameters into account (e.g., time point of testing, the cognitive function that is to be tested, and who is tested). ${ }^{42,43}$ Surprisingly, there are almost no guidelines or guidelines remain vague on the content and frequency of cognitive assessment in the field of ECT. ${ }^{43-47}$

Moreover, up to now, researchers in our field have used neuropsychological tests that were developed in other research fields and diagnostic categories and were not originally designed to capture ECT-related cognitive problems. We still do not know exactly which tests are both valid and sensitive enough to capture the cognitive side effects of ECT. For example, there is still no consensus on which of the existing autobiographical memory tests should be used for ECT research and clinical practice. ${ }^{48,49}$ As a result, the precise extent of the impact of ECT on autobiographical memory and other cognitive domains remains unclear. 


\section{MMSE Changes During and After ECT in Late-Life Depression}

The use of modern techniques, such as an iPadbased measurement approach or interactive voice response, could be future solutions in finding a balance between easy-to-use and sensitive tests for monitoring cognitive effects of ECT in the elderly. ${ }^{50,51}$

In conclusion, our data offer no evidence for ECTrelated deleterious cognitive effects, as measured with the MMSE, during and up to 6 months after the index course of ECT in patients with LLD. In the event of baseline cognitive impairment, MMSE scores seem to improve significantly during ECT and 6 months after ending it. Our findings, although based on a global measure such as the MMSE, contradict the assumption that older patients with an impaired cognitive function before the start of ECT are more vulnerable for developing ECT-related cognitive side effects. Further study on the development of neuropsychological tests that are specially designed to detect cognitive effects of ECT and are tolerable for older ECT patients with a severe depression is warranted.

The authors have no financial conflicts or other conflict of interest to disclose.

\section{References}

1. Schulz R, Drayer RA, Rollman BL: Depression as a risk factor for non-suicide mortality in the elderly. Biol Psychiatry 2002; 52:205-225

2. Erlangsen A, Zarit SH, Tu X, et al: Suicide among older psychiatric inpatients: an evidence-based study of a high-risk group. Am J Geriatr Psychiatry 2006; 14:734-741

3. Luppa M, König H, Heider D, et al: Direct costs associated with depressive symptoms in late life: a 4.5 year prospective study. Int Psychogeriatr 2013; 25:292-302

4. Zivin $\mathrm{K}$, Wharton $\mathrm{T}$, Rostant $\mathrm{O}$ : Economic, public health, and caregiver burden of late-life depression. Psychiatr Clin North Am 2013; 36:631-649

5. Van Diermen L, van den Ameele S, Kamperman AM, et al: Prediction of electroconvulsive therapy response and remission in major depression: meta-analysis. Br J Psychiatry 2018; 212:71-80

6. Haq AU, Sitzmann AF, Goldman ML, et al: Response of depression to electroconvulsive therapy: a meta-analysis of clinical predictors. J Clin Psychiatry 2015; 76:1374-1384

7. Zervas IM, Calev A, Jandorf L, et al: Age-dependent effects of electroconvulsive therapy on memory. Convuls Ther 1993; 9:39-42

8. Tielkes CE, Comijs HC, Verwijk E, et al: The effects of ECT on cognitive functioning in the elderly: a review. Int J Geriatr Psychiatry $2008 ; 23: 789-795$

9. Gardner BK, O'Connor DW: A review of the cognitive effects of electroconvulsive therapy in older adults. J ECT 2008; 24:68-80

10. Kumar S, Mulsant BH, Liu AY, et al: Systematic review of cognitive effects of electroconvulsive therapy in late-life depression. Am J Geriatr Psychiatry 2016; 24:547-565

11. Semkovska M, McLoughlin DM: Objective cognitive performance associated with electroconvulsive therapy for depression: a systematic review and meta-analysis. Biol Psychiatry 2010; 68: 568-577

12. Semkovska M, Keane D, Babalola $\mathrm{O}$, et al: Unilateral brief-pulse electroconvulsive therapy and cognition: effects of electrode placement, stimulus dosage and time. J Psychiatr Res 2011; 45:770-780

13. Verwijk E, Comijs HC, Kok RM, et al: Short- and long-term neurocognitive functioning after electroconvulsive therapy in depressed elderly: a prospective naturalistic study. Int Psychogeriatr 2014; 26:315-324

14. Schatzberg AF, Posener JA, Debattista C, et al: Neuropsychological deficits in psychotic versus nonpsychotic major depression and no mental illness. Am J Psychiatry 2000; 157:1095-1100
15. Bosboom P, Deijen JB: Age-related cognitive effects of ECT and ECT-induced mood improvement in depressive patients. Depress Anxiety 2006; 23:93-101

16. Bayless JD, McCormick LM, Brumm MC, et al: Pre- and post-electroconvulsive therapy multidomain cognitive assessment in psychotic depression: relationship to premorbid abilities and symptom improvement. J ECT 2010; 26:47-52

17. Folstein MF, Folstein SE, McHugh PR: "Mini-mental state". A practical method for grading the cognitive state of patients for the clinician. J Psychiatr Res 1975; 12:189-198

18. Rajii TK, Miranda D, Mulsant BH, et al: The MMSE is not an adequate screening cognitive instrument in studies of late-life depression. J Psychiatr Res 2009; 43:464-470

19. Anthony JC, LeResche L, Niaz U, et al: Limits of the 'Mini-Mental State' as a screening test for dementia and delirium among hospital patients. Psychol Med 1982; 12:397-408

20. O'Connor DW, Pollitt PA, Hyde JB, et al: The reliability and validity of the Mini-Mental State in a British community survey. J Psychiatr Res 1989; 23:87-96

21. Rubin EH, Kinscherf DA, Figiel GS, et al: The nature and time course of cognitive side effects during ECT in the elderly. J Geriatr Psychiatry Neurol 1993; 6:78-83

22. Rosen J, Mulsant BH, Nebes RD: A pilot study of interictal cognitive changes in elderly patients during ECT. Int J Geriatr Psychiatry 1992; 7:407-410

23. Oudega ML, van Exel E, Wattjes MP, et al: White matter hyperintensities and cognitive impairment during electroconvulsive therapy in severely depressed elderly patients. Am J Geriatr Psychiatry $2014 ; 22: 157-166$

24. Hausner L, Damian M, Sartorius A, et al: Efficacy and cognitive side effects of electroconvulsive therapy (ECT) in depressed elderly inpatients with coexisting mild cognitive impairment or dementia. J Clin Psychiatry 2011; 72:91-97

25. Stoudemire A, Hill CD, Morris R, et al: Long-term affective and cognitive outcome in depressed older adults. Am J Psychiatry $1993 ; 150: 896-900$

26. Dols A, Bouckaert F, Sienaert P, et al: Early- and late-onset depression in late life: a prospective study on clinical and structural brain characteristics and response to electroconvulsive therapy. Am J Geriatr Psychiatry 2017; 25:178-189

27. American Psychiatric Association: Diagnostic and statistical manual of mental disorders. Washington, DC, American Psychiatric Press, 2000 
28. Montgomery SA, Åsberg M: A new depression scale designed to be sensitive to change. Br J Psychiatry 1979; 134:382-389

29. Sheehan DV, Lecrubier Y, Sheehan KH, et al: The Mini-International Neuropsychiatric Interview (M.I.N.I.): the development and validation of a structured diagnostic psychiatric interview for DSM-IV and ICD-10. J Clin Psychiatry 1998; 59:22-33

30. Obbels J, Verwijk E, Vansteelandt K, et al: Long-term neurocognitive functioning after electroconvulsive therapy in patients with late-life depression. Acta Psychiatr Scand 2018; 138:223-231

31. Lezak MD, Howieson DB, Loring DW, et al: Neuropsychological Assessment. New York, Oxford University Press, 2004

32. Verbeke G, Molenberghs G: Linear Mixed Models for Longitudinal Data. New York, Springer, 2000

33. Naumova EN, Must A, Laird NM: Tutorial in biostatistics: evaluating the impact of 'critical periods' in longitudinal studies of growth using piecewise mixed effects models. Int $\mathrm{J}$ Epidemiol 2001; 30:1332-1341

34. Little RJA, Rubin DB: Statistical Analysis With Missing Data. New York, Wiley, 2002

35. Becker BJ: Synthesizing standardized mean-change measures. Br J Math Stat Psychol 1988; 41:257-278

36. Lipsey MW, Wilson DB: Practical Meta-Analysis. London, Sage Publications, 2000

37. Cohen J: Statistical Power Analysis in the Behavioral Sciences. Hillsdale, Erlbaum, 1988

38. SAS Institute Inc: SAS/QC 9.3 User's Guide. Cary, NC, SAS Institute Inc, 2011

39. Oudega ML, Dols A, Adelerhof I, et al: Contribution of white matter hyperintensities, medial temporal lobe atrophy and cortical atrophy on outcome, seven to twelve years after ECT in severely depressed geriatric patients. J Affect Disord 2015; 185:144-148

40. O'Connor DW, Gardner B, Eppingstall B, et al: Cognition in elderly patients receiving unilateral and bilateral electroconvulsive therapy: a prospective, naturalistic comparison. J Affect Disord $2010 ; 124: 235-240$
41. Moirand R, Galvao F, Lecompte M, et al: Usefulness of the Montreal Cognitive Assessment (MoCA) to monitor cognitive impairments in depressed patients receiving electroconvulsive therapy. Psychiatry Res 2018; 259:476-481

42. Porter RJ, Douglas K, Knight RG: Monitoring of cognitive effects during a course of electroconvulsive therapy: recommendations for clinical practice. J ECT 2008; 24:25-34

43. Rasmussen KG: What type of cognitive testing should be part of routine electroconvulsive therapy practice? J ECT 2016; 32:7-12

44. Sienaert P, De Fruyt J, Dierick M: Electroconvulsietherapie: Aanbevelingen Voor de Praktijk. Gent, Academia Press, 2006

45. Van den Broek WW, Birkenhäger TK, de Boer JP, et al: Richtlijn Electroconvulsietherapie. Utrecht, De Tijdstroom, 2010

46. American Psychiatric Association: The Practice of Electroconvulsive Therapy: Recommendations for Treatment, Training, and Privileging. Washington, DC, American Psychiatric Association, 2001

47. Milev RV, Giacobbe P, Kennedy SH, et al: Canadian Network for Mood and Anxiety Treatments (CANMAT) 2016 clinical guidelines for the management of adults with major depressive disorder: section 4. Neurostimulation treatments. Can J Psychiatry 2016; 61:561-575

48. Sackheim HA: Autobiographical memory and electroconvulsive therapy: do not throw out the baby. J ECT 2014; 30:177-186

49. Semkovska M, McLoughlin DM: Retrograde autobiographical amnesia after electroconvulsive therapy: on the difficulty of finding the baby and clearing murky bathwater. J ECT 2014; 30:187-188

50. Müller HHO, Reike M, Grosse-Holz S, et al: Electroconvulsive therapy hasn't negative effects on short-term memory function, as assessed using a bedside hand-held device. Ment Illn 2017; 9:30-35

51. Fazzino TL, Rabinowitz T, Althoff RR, et al: Monitoring daily affective symptoms and memory function using interactive voice response in outpatients receiving electroconvulsive therapy. J ECT 2013; 29:318-324 